\title{
Port-Hamiltonian based Optimal Power Flow algorithm for multi-terminal DC networks
}

\author{
Ernest Benedito $^{\mathrm{a}}$, Dunstano del Puerto-Flores ${ }^{\mathrm{b}}$, Arnau Dòria-Cerezo ${ }^{\mathrm{a}, *}$, \\ Jacquelien M.A. Scherpen ${ }^{c}$ \\ ${ }^{a}$ Inst. of Industrial and Control Engineering, Universitat Politècnica de Catalunya, \\ Barcelona (Spain) \\ ${ }^{b}$ Dept. of Mechanical-Electrical Engineering, University of Guadalajara, Guadalajara \\ (Mexico) \\ ${ }^{c}$ Engineering and Technology Institute, University of Groningen, Groningen (The \\ Netherlands)
}

\begin{abstract}
In this paper an algorithm for solving the Optimal Power Flow problem for multi-terminal DC networks based on the gradient method is proposed. The aim is seeking the optimal point subject to voltage, current and power constraints. The algorithm is described by a continuous-time port-Hamiltonian model, and the inequality constrains are included by the use of barrier functions. The dynamics of the algorithm is studied and stability conditions are obtained. Finally, the method is used for the offshore wind integration grid in the North Sea and the interconnection with the network dynamics is tested by means of numerical simulations.
\end{abstract}

Keywords: Optimal power flow, port-Hamiltonian systems, gradient method, DC networks, cyclic networks

\section{Introduction}

Multi-terminal DC (MTDC) networks emerged as important systems for the transmission and distribution of electrical energy, from low voltage applications such as household DC networks, [1, to high voltage systems, like High-Voltage 5 DC (HVDC) networks, 2, including spacecraft systems, data centers, traction power systems, plug-in electric vehicles, DC microgrids involving PV generation and battery storage, or shipboard power systems, see 3 and references therein. Usually, the control of MTDC networks involves a hierarchical structure, with a decentralized controller which acts locally in each node of the network and an upper level supervisor algorithm, known as Optimal Power Flow (OPF),

${ }^{*}$ Corresponding author: Av. Diagonal 647, 08028 Barcelona (Spain). Phone num: +34.934016659 , Fax num: +34.934016605,

Email address: arnau.doria@upc.edu (Arnau Dòria-Cerezo) 
that provides the voltage references to each node to achieve the desired (and optimal) energy transmission among the nodes, where some voltages, currents and powers are subjected to equality and inequality constraints.

Many references can be found related to the local control of the voltage nodes 15 and its associated voltage source converter (VSC). Papers included vary from the well known droop-control technique (see examples in [4], [5], and [6]), to control strategies using advanced control methods, such as $H_{\infty}$ robust control [7, model predictive control [8-[9], passivity-based techniques [10]-[11, sliding modes [12] or complex networks approaches [13].

20 However, the OPF problem for MTDC networks is still an open problem. Despite the analogies between $\mathrm{AC}$ and DC OPF problems, the main difference is the optimization target; frequency in $\mathrm{AC}$, voltage in $\mathrm{DC}, 14$, thus $\mathrm{OPF}$ algorithms for AC methods are not suitable for DC problems. Usually OPF in DC networks is solved via standard methods (both deterministic and heuristic) 25 such as interior point algorithms with barrier functions [15], covariance matrix adaption evolutionary strategy (CMA-ES) [16, or tools available in engineering softwares such as RT-Lab [17] or the Optimization Toolbox of Matlab [18]-[19].

In general, the OPF algorithm is implemented in a microprocessor that provides the solution after a several iterations. This means that the optimal soso lution is available at certain time values. Alternatively, the OPF problem can be solved using a continuous-time algorithm [20]-21. On another hand, only few references study the stability of the algorithms for solving the OPF problem, see [22]-23]. The stability analysis is necessary because, usually, the OPF implies the minimization of a cost (loss) function that, expressed in terms of 35 voltages, depends on the weighted Laplacian matrix. Since this matrix is positive semidefinite, the function turns to be just convex [22, and not strictly convex.

In this paper, an algorithm based on the primal-dual gradient method is proposed, that, in opposite to other methods, allows a simple and continuous time 40 mathematical description as a dynamical system. Moreover, the advantages of using a continuous-time algorithm are twofold: firstly, stability of the gradient method dynamics can be analyzed using Lyapunov methods and, secondly, the optimization algorithm can be easily interconnected with the network, providing stability of the whole dynamics. The use of the continuous-time gradient 45 method to find the optimal point has been studied [24, 25], and stability problems may appear when the cost function is no strictly convex. Modifications of the problem statement can overcome the stability issue, where the Krasovskii method is used to prove stability, see e.g. [26]. Alternatively, the LaSalle Invariance Principle can be used to guarantee the stability for a certain combination of so cost functions and constraints in 21, 225. Recently, the continuous-time portHamiltonian description of the gradient method has been proposed in [20] 27, which results have motivated this work.

This paper extends the results presented in [20, where a continuous-time primal-dual gradient method for solving the OPF problem in MTDC networks 55 was studied. In [20] only problems with equality constraints were studied, but inequality constraints need to be considered in a realistic case. The main con- 
tributions of the present paper are: i) describing the method for solving an optimization problem with inequality constraints as a port-Hamiltonian system, with the use of barrier functions, ii) providing stability conditions of the method, and iii) its application to a practical case (the North Sea wind integration grid), providing a faster regulation to the optimal point and a better performance than classical discrete-time OPF implementations.

The paper is organized as follows. Preliminaries and definitions are given in Section 2, The port-Hamiltonian representation of the primal-dual gradient

65 method is described in Section 3, and its application to the OPF for resistive networks is detailed in Section 4. In Section 5 the numerical results for the North Sea network are presented and, finally, the Conclusions are stated in Section 6 .

\section{Preliminaries and definitions}

In this paper a resistive DC network is considered: an undirected, connected, and weighted graph, $\mathcal{G}$, with $n$ nodes (vertices) and $m$ branches (edges). The following results are obtained from classical graph theory books [28].

Definition 1. $1 \in \mathbb{R}^{n}$ is the vector consisting of only ones.

Definition 2 (Incidence matrix). Consider an arbitrary orientation of the edges. The (vertex-edge) incidence matrix, $\boldsymbol{B} \in \mathbb{R}^{n \times m}$, is defined by the ( $\left.k, l\right)$ th elements as

$$
\boldsymbol{b}_{k l}=\left\{\begin{aligned}
1 & \text { if the vertex } k \text { is the head of edge } l \\
-1 & \text { if the vertex } k \text { is the tail of edge } l \\
0 & \text { otherwise. }
\end{aligned}\right.
$$

Definition 3 (Laplacian matrix). The weighted Laplacian matrix is defined as

$$
\boldsymbol{W}=\boldsymbol{B} \boldsymbol{G} \boldsymbol{B}^{T},
$$

where $\boldsymbol{G}$ is the $m \times m$ diagonal matrix with the weights of each edge [29].

From the definitions above, the following properties are satisfied:

75 P1. $\operatorname{ker}\left(\boldsymbol{B}^{\boldsymbol{T}}\right)=\{\alpha \mathbf{1} \mid \alpha \in \mathbb{R}\}$, then $\boldsymbol{B}^{T} \mathbf{1}=0 . \operatorname{rank}\left(\boldsymbol{B}^{T}\right)=n-1$.

P2. The Laplacian matrix, $\boldsymbol{W}$, has zero row-sum: $\sum_{j} \boldsymbol{w}_{k j}=0, k=1, \ldots, n$

P3. $\boldsymbol{1}$ is a right eigenvector of $\boldsymbol{W}$ with eigenvalue 0 , i.e., $\boldsymbol{W} \mathbf{1}=0$.

Remark 1. The Kirchhoff's Current Law (KCL) in a circuit with external current sources naturally arises from the incidence matrix as

$$
\boldsymbol{B} i=i_{\text {ext }},
$$

where $i \in \mathbb{R}^{m}$ are the currents through the branches (edges), and $i_{\text {ext }} \in \mathbb{R}^{n}$ are the injected currents at the nodes (vertices). 


\section{Port-Hamiltonian representation of the constrained gradient method}

\subsection{Equality constraint case}

The stability of the gradient method for strictly convex functions was already studied in [30]. Recently, the stability analysis has been done using passive systems properties in [27] and 25, which entails a different perspective

85 that becomes very useful for interconnecting systems, see [31] as example. In this subsection, the port Hamiltonian representation of the gradient method algorithm presented in 27] and 25] is presented.

Consider the minimization problem defined by

$$
\begin{array}{cl}
\min _{x} & f(x) \\
\text { s.t. } & \boldsymbol{A} x-b=0,
\end{array}
$$

where $x \in \mathbb{R}^{n}, f: \mathbb{R}^{n} \rightarrow \mathbb{R}$ is a convex function, $\boldsymbol{A} \in \mathbb{R}^{p \times n}$ and $b \in \mathbb{R}^{p}$. The optimal value of (4)-(5) can be obtained finding the saddle-point of the Lagrangian

$$
\mathcal{L}(x, \lambda)=f(x)+\lambda^{T}(\boldsymbol{A} x-b)
$$

where $\lambda \in \mathbb{R}^{p}$. The gradient method for finding the saddle-point of $(6)$ is

represented by the following system of differential equations:

$$
\begin{aligned}
& \boldsymbol{T}_{x} \dot{x}=-\nabla f(x)-\boldsymbol{A}^{T} \lambda \\
& \boldsymbol{T}_{\lambda} \dot{\lambda}=\boldsymbol{A} x-b
\end{aligned}
$$

and the port-Hamiltonian representation of the gradient method is given by

$$
\dot{z}=\left(\begin{array}{cc}
0 & -\boldsymbol{A}^{T} \\
\boldsymbol{A} & 0
\end{array}\right) \nabla H-\left(\begin{array}{c}
\nabla f(x) \\
b
\end{array}\right)
$$

where $z=\left(\boldsymbol{T}_{x} x, \boldsymbol{T}_{\lambda} \lambda\right)$ and $\boldsymbol{T}_{x}, \boldsymbol{T}_{\lambda}>0$ are symmetric positive definite matrices, and can be used to tune the convergence time to the solution. The Hamiltonian function is given by

$$
H=\frac{1}{2} z^{T} \boldsymbol{T}^{-1} z
$$

where $\boldsymbol{T}=\operatorname{blockdiag}\left(\boldsymbol{T}_{x}, \boldsymbol{T}_{\lambda}\right)$, and the $\nabla(\cdot)$ operator is used for the gradient (as a column vector).

Let us define $z^{*}=\left(\boldsymbol{T}_{x} x^{*}, \boldsymbol{T}_{\lambda} \lambda^{*}\right)$ as the (unique) equilibrium point of $(9)$ and the shifted Hamiltonian by

$$
H^{*}=\frac{1}{2}\left(z-z^{*}\right)^{T} \boldsymbol{T}^{-1}\left(z-z^{*}\right)
$$

then $(9)$ is equivalent to

$$
\dot{z}=\left(\begin{array}{cc}
0 & -\boldsymbol{A}^{T} \\
\boldsymbol{A} & 0
\end{array}\right) \nabla H^{*}-\left(\begin{array}{c}
\nabla f(x)-\nabla f\left(x^{*}\right) \\
0
\end{array}\right) .
$$

The asymptotic stability of 9 can be proved under the following conditions. 
Proposition 1. Assume that $z^{*}$ is a (unique) equilibrium point of $(9), \operatorname{ker}\left(\boldsymbol{A}^{T}\right)=$ totically to $z^{*}$, i.e., $(x, \lambda) \rightarrow\left(x^{*}, \lambda^{*}\right)$.

Proof. (From [27]) The time derivative of the shifted Hamiltonian is

$$
\dot{H}^{*}=-\left(x-x^{*}\right)^{T}\left(\nabla f(x)-\nabla f\left(x^{*}\right)\right) \leq 0,
$$

since $f(x)$ is convex, and the equality holds if and only if $x=x^{*}$ since $f(x)$ is strictly convex. Using LaSalle's invariant principle, on the largest invariant set where $\dot{H}^{*}=0$, one has that $\lambda=\lambda^{*}$ as $\boldsymbol{A}^{T}\left(\lambda-\lambda^{*}\right)=0$, which proves the proposition.

\subsection{Equality and inequality constraint case}

Consider now the constrained minimization problem [32, defined by

$$
\begin{array}{cl}
\min _{x} & f(x) \\
\text { s.t. } & \boldsymbol{A} x-b=0, \\
& g_{j}(x) \leq 0 \quad j=1, \ldots, p
\end{array}
$$

where $x \in \mathbb{R}^{n}, f: \mathbb{R}^{n} \rightarrow \mathbb{R}$ and $g_{j}: \mathbb{R}^{n} \rightarrow \mathbb{R}, \forall j$, are convex functions, $\boldsymbol{A} \in \mathbb{R}^{q \times n}$ and $b \in \mathbb{R}^{q}$.

It has been proved that the gradient method applied to the minimization problem with inequality constraints (14)-(16) results in a passive dynamical system [25] and 21], but it can not be represented in the port-Hamiltonian framework. In this paper, the use of barrier functions for the inequality constraints which does allow the port-Hamiltonian representation has been proposed. The use of barrier functions approximates the solution of the problem defined by (14)-(16) guaranteeing that the solution fulfils (16). The accuracy of the approximation can be properly adjusted, see details in [32. This approach needs that the initial conditions must be in the domain defined by the inequality constraints.

115 The proposed solution consists of rewriting the inequality constraint in the objective as follows

$$
\begin{array}{ll}
\min _{x} & f(x)+\sum_{j=1}^{p} c\left(g_{j}(x)\right) \\
\text { s.t. } & \boldsymbol{A} x-b=0
\end{array}
$$

where $c$ is a function that ideally is 0 when $g_{j} \leq 0$ and $\infty$ if $g_{j}>0$. An approximation is the logarithmic barrier function,

$$
c(u)=-k \log (-u)
$$

where $k$ is a parameter that can be used to set the accuracy of the approximation. $c(u)$, defined in 197 , is a convex and non decreasing function, hence, the new 


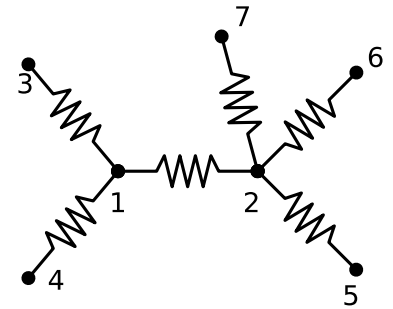

a)

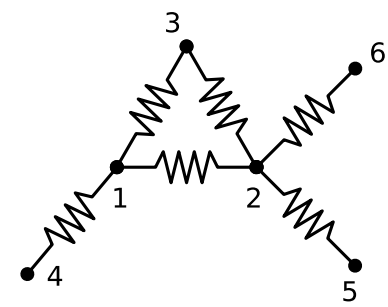

b)

Figure 1: Resistive circuits examples: a) acyclic resistive network, and b) cyclic resistive network.

objective function (17) is convex. Moreover, 17) is strictly convex if $f(x)$ is strictly convex.

Then, the port-Hamiltonian system description of the method is

$$
\dot{z}=\left(\begin{array}{cc}
0 & -\boldsymbol{A}^{T} \\
\boldsymbol{A} & 0
\end{array}\right) \nabla H-\left(\begin{array}{c}
\nabla f(x)+\nabla \gamma(x) \\
b
\end{array}\right)
$$

where $\gamma(x)=\sum_{j=1}^{p} c\left(g_{j}(x)\right)$. Note that if $f(x)$ is strictly convex Proposition 1 can be used.

\section{Port-Hamiltonian based gradient method applied to the OPF for DC networks: stability analysis}

Before presenting the OPF problem, let us first introduce the DC network. Consider a resistive DC network with $n$ nodes and $m$ branches, with $m$ resistors $R_{l}>0$ associated to each branch, $l=1, \ldots m$, and one voltage source in each node, $v_{k}$ where $k=1, \ldots, n$. See two kind of resistive circuits; acyclic and cyclic, in Figure 1 .

From Kirchhoff's and Ohm's laws, the voltages (at each node) are related with the currents (through each resistor) by

$$
\boldsymbol{B}^{T} v=\boldsymbol{R} i
$$

where $v \in \mathbb{R}^{n}$ and $i \in \mathbb{R}^{m}$, are the voltage and current vectors, respectively, $\boldsymbol{B}$ is the incidence matrix of the network, and $\boldsymbol{R}=\operatorname{diag}\left(R_{l}\right)>0$.

The control problem consists of finding an optimal voltage vector $v^{\text {opt }}$ that minimizes the losses by Joule's effect, with some constraints on the voltages. The network losses function is the sum of the losses in all resistors, $f(i)=\sum_{l=1}^{m} R_{l} i_{l}^{2}$, in a matrix form

$$
f(i)=i^{T} \boldsymbol{R} i .
$$

From the conductance of the $l$-branch, $G_{l}=\frac{1}{R_{l}}$, the conductance matrix can be defined as $\boldsymbol{G}=\boldsymbol{R}^{-1}$, and using (21), the cost function yields in terms of the weighted Laplacian as in Definition 3

$$
f(v)=v^{T} \boldsymbol{W} v,
$$


where (2) has been used.

Remark 2. Note that, from Definition 3 and Property 2, the weighted Laplacian, $\boldsymbol{W}$, is positive semidefinite but it is not positive definite. Then, the loss function $f(v)$ in $\sqrt{23})$ is not strictly convex.

Now one can formulate the OPF problem of the setting, i.e., assuming that the node voltages are linearly constrained, the OPF problem can be defined as

$$
\begin{array}{cl}
\min _{v} & f(v)=v^{T} \boldsymbol{W} v \\
\text { s.t. } & \boldsymbol{A} v-b=0 \\
& \boldsymbol{Q} v-d \leq 0
\end{array}
$$

where $b \in \mathbb{R}^{q}$ and $d \in \mathbb{R}^{p}$ refer to the desired values, and the constraints on the node voltages, respectively, and $\boldsymbol{A}, \boldsymbol{Q}$ are matrices relating the voltages, $v$, with their desired, maximum or minimum values.

Following the approximation described in Section 3.2 , with the barrier function of (19), the OPF problem can be approximated by

$$
\begin{array}{cl}
\min _{v} & f_{c}(v)=v^{T} \boldsymbol{W} v+\gamma(v) \\
\text { s.t. } & \boldsymbol{A} v-b=0
\end{array}
$$

with

$$
\gamma(v)=-\sum_{j=1}^{p} k_{j} \log \left(-[\boldsymbol{Q}]_{j} v+d_{j}\right)
$$

and $[\cdot]_{j}$ refers to the $j$-th row of matrix $(\cdot)$.

Proposition 2. $f_{c}(v)$ defined in (27) and (29) is a strictly convex function if and only if there exists a $j \in\{1, \ldots, p\}$ such that $[\boldsymbol{Q}]_{j} \mathbf{1} \neq 0$.

Proof. As the Hessian of $f_{c}(v)$, namely $\nabla^{2} f_{c}(v)$, exists at each point in $\operatorname{dom}\left(\mathrm{f}_{c}\right)$, $f_{c}$ is strictly convex if and only if $\nabla^{2} f_{c}(v)$ is positive definite.

Let $v \in \operatorname{dom}\left(\mathrm{f}_{c}\right)$. Then $\nabla^{2} f_{c}(v)$ can be computed as follows

$$
\nabla^{2} f_{c}(v)=2 \boldsymbol{W}+\sum_{j=1}^{p} k_{j} \frac{[\boldsymbol{Q}]_{j}^{T} \cdot[\boldsymbol{Q}]_{j}}{\left([\boldsymbol{Q}]_{j} v-d_{j}\right)^{2}}
$$

Suppose that $[\boldsymbol{Q}]_{j} \mathbf{1}=0, \forall j$. Then $\nabla^{2} f_{c}(v)$ is not positive definite as $\mathbf{1}^{T} \nabla^{2} f_{c}(v) \mathbf{1}=$ 0 . Conversely, supose that $[\boldsymbol{Q}]_{\alpha} \mathbf{1} \neq 0$ with $\alpha \in\{1, \ldots, p\}$. Then, $\nabla^{2} f_{c}(v)$ is positive definite as

$$
r^{T} \boldsymbol{W} r>0, \quad \forall r \in \mathbb{R}^{n} \backslash \operatorname{Span}(\mathbf{1})
$$

and

$$
r^{T} \frac{[\boldsymbol{Q}]_{\alpha}^{T} \cdot[\boldsymbol{Q}]_{\alpha}}{\left([\boldsymbol{Q}]_{\alpha} v-d_{\alpha}\right)^{2}} r>0, \quad \forall r \in \operatorname{Span}(\mathbf{1}) \backslash\{0\} .
$$


Remark 3. Note that if the vector $d$ in 26) refers to one single value for a node, the corresponding row in $\boldsymbol{Q}$ has all zeros except one 1. Consequently, from Proposition 2, $f_{c}(v)$ is strictly convex.

The optimization algorithm in port-Hamiltonian form 20 applied to the OPF problem described by 27)-28 is given by

$$
\dot{z}=\left(\begin{array}{cc}
-\boldsymbol{W} & -\boldsymbol{A}^{T} \\
\boldsymbol{A} & 0
\end{array}\right) \nabla H-\left(\begin{array}{c}
\nabla \gamma(v) \\
b
\end{array}\right)
$$

where $z=\left(\boldsymbol{T}_{v} v, \boldsymbol{T}_{\lambda} \lambda\right)$ and $\boldsymbol{T}_{v}=\boldsymbol{T}_{v}^{T}, \boldsymbol{T}_{\lambda}=\mathbf{T}_{\lambda}^{\mathbf{T}}$ are positive definite matrices and $\lambda \in \mathbb{R}^{q}$. The Hamiltonian function is given by

$$
H=\frac{1}{2} z^{T} \boldsymbol{T}^{-1} z
$$

where $\boldsymbol{T}=\operatorname{blockdiag}\left(\boldsymbol{T}_{v}, \boldsymbol{T}_{\lambda}\right)$.

Let us define $z^{*}=\left(\boldsymbol{T}_{v} v^{*}, \boldsymbol{T}_{\lambda} \lambda^{*}\right)$ as an equilibrium point of (31) and the shifted Hamiltonian by

$$
H^{*}=\frac{1}{2}\left(z-z^{*}\right)^{T} \boldsymbol{T}^{-1}\left(z-z^{*}\right)
$$

then 31 is equivalent to

$$
\dot{z}=\left(\begin{array}{cc}
-\boldsymbol{W} & -\boldsymbol{A}^{T} \\
\boldsymbol{A} & 0
\end{array}\right) \nabla H^{*}-\left(\begin{array}{c}
\nabla \gamma(v)-\nabla \gamma\left(v^{*}\right) \\
0
\end{array}\right)
$$

Similar to Proposition 1 the asymptotic stability of 31 can be proved under the following conditions.

Proposition 3. Assume that $z^{*}$ is an equilibrium point of (31), and one of the two following conditions hold

C1: if exists $j \in\{1, \ldots, p\}$ such that $[\boldsymbol{Q}]_{j} \mathbf{1} \neq 0$,

C2: $\left(\boldsymbol{T}_{v}^{-1} \boldsymbol{A}^{T} \boldsymbol{T}_{\lambda}^{-1} \boldsymbol{A}\right) \cdot \mathbf{1}$ not in $\operatorname{Span}(\mathbf{1})$.

Then, the dynamics in (31) will converge asymptotically to $z^{*}$, i.e., $(v, \lambda) \rightarrow$ $160\left(v^{*}, \lambda^{*}\right)$.

Proof. The time derivative of the shifted Hamiltonian 33 with 34 is

$$
\begin{aligned}
\dot{H}^{*} & =\left(z-z^{*}\right)^{T} \boldsymbol{T}^{-1}\left[\left(\begin{array}{cc}
-\boldsymbol{W} & \boldsymbol{A}^{T} \\
\boldsymbol{A} & 0
\end{array}\right) \boldsymbol{T}^{-1}\left(z-z^{*}\right)-\left(\begin{array}{c}
\nabla \gamma(v)-\nabla \gamma\left(v^{*}\right) \\
0
\end{array}\right)\right] \\
& =-\left(v-v^{*}\right)^{T} \boldsymbol{W}\left(v-v^{*}\right)-\left(v-v^{*}\right)^{T}\left(\nabla \gamma(v)-\nabla \gamma\left(v^{*}\right)\right) \leq 0 .
\end{aligned}
$$

If condition $\mathrm{C} 1$ is fulfilled, $\dot{H}^{*}<0$ is verified from Proposition 2 On the other hand, if only $\mathrm{C} 2$ holds, one has that since $\boldsymbol{W}$ is positive semidefinite, and 
Demand curves,

forecast, network...

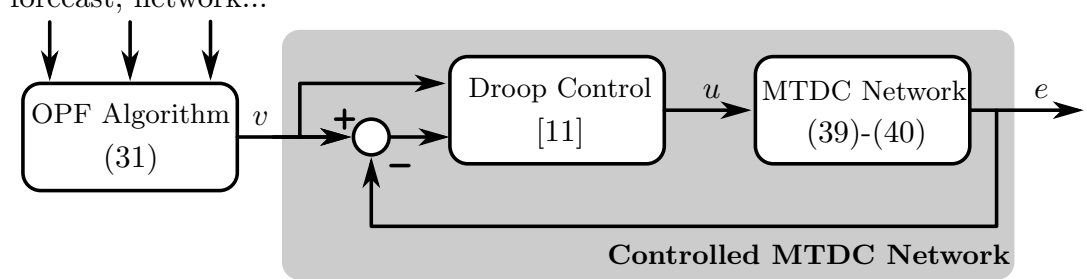

Figure 2: Supervision and control scheme for a multi-terminal DC network.

consequently the equality holds if and only if $v-v^{*} \in \operatorname{ker}(\boldsymbol{W})$, i.e., $v-v^{*}=a \mathbf{1}$ with $a \in \mathbb{R}$.

On the largest invariant set where $\dot{H}^{*}=0$, one has that

$$
\ddot{a} \mathbf{1}=-a \boldsymbol{T}_{v}^{-1} \boldsymbol{A}^{T} \boldsymbol{T}_{\lambda}^{-1} \boldsymbol{A} \mathbf{1} .
$$

Then $a=0$ as $\left(\boldsymbol{T}_{v}^{-1} \boldsymbol{A}^{T} \boldsymbol{T}_{\lambda}^{-1} \boldsymbol{A}\right) \cdot \mathbf{1}$ not in $\operatorname{Span}(\mathbf{1})$, and $v=v^{*}$. Using LaSalle's invariance principle, in the set one has that $\lambda=\lambda^{*}$ as

$$
\boldsymbol{A}^{T}\left(\lambda-\lambda^{*}\right)=0
$$

and $\operatorname{ker}\left(\boldsymbol{A}^{T}\right)=\{0\}$, proving the proposition.

\section{The port-Hamiltonian OPF applied to a multi-terminal DC net- work}

In this section, the OPF algorithm proposed in the previous section is applied to a controlled multi-terminal DC network with $n$ nodes. Figure 2 shows the classical hierarchical control scheme consisting of:

i) a supervisor algorithm (OPF Algorithm) that sets the required voltages, $v=\left(v_{1}, \ldots, v_{n}\right)$, to all network nodes optimising a cost function and constraints that depend on the market demand, forecast, and network parameters,

ii) a voltage control (Droop Control) algorithm with the aim to regulate the voltage nodes, $e=\left(e_{1}, \ldots, e_{n}\right)$, to the reference values calculated by the OPF, $v$, by injecting current, $u=\left(u_{1}, \ldots, u_{n}\right)$, through the VSCs (the VSC control is out of the focus of this paper), and

iii) the network dynamics (MTDC Network).

The dynamical model and the OPF problem for a multi-terminal DC network are presented in the following subsections, and the example of the North Sea offshore wind integration network is numerically tested in Section 5.3 . 


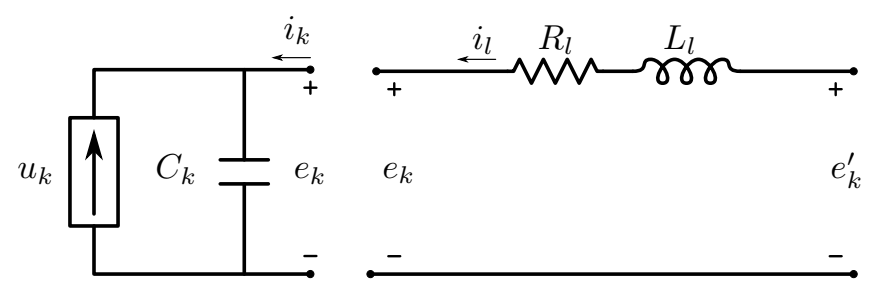

Figure 3: Equivalent circuits: VSC (left); transmission line (right).

Remark 4. In this paper, a perfect knowledge of the grid parameters is assumed. Parametric uncertainties will imply a non-minimal solution that could also break some of the equality/inequality constraints. This non-optimal solution could imply oscillations in the power network as was studied in [11]. To prevent this problem, a parameter estimator of the line resistances or an adaptive scheme can be used.

\subsection{Dynamical model and control of a multi-terminal DC network}

Multi-terminal DC networks are usually modelled as circuits with RL lines and current sources in parallel with capacitors representing the voltage source converters (VSC). Figure 3 shows the equivalent circuits for both VSC and lines, where $u_{k}$ is the current injected by the power converters (that act as control inputs for the DC network), $C_{k}$ is the capacitance at node $k$, and $R_{l}, L_{l}$ are the lumped values for the resistance and inductance for the line connecting nodes $k$ and $l$. In this section, $e_{k}$ is adopted for the voltages at the node to not confuse with the values used for the OPF algorithm.

The dynamics of multi-terminal DC networks can be written in a compact form [13], as

$$
\begin{aligned}
\boldsymbol{C} \dot{e} & =-\boldsymbol{B} i+u \\
\boldsymbol{L} \dot{i} & =-\boldsymbol{R} i+\boldsymbol{B}^{T} e
\end{aligned}
$$

where $e \in \mathbb{R}^{n}$ contain all the node/capacitor voltages, $i \in \mathbb{R}^{m}$ the line/inductor currents, $\boldsymbol{C} \in \mathbb{R}^{n \times n}$ is the capacitor matrix, $\boldsymbol{L}, \boldsymbol{R} \in \mathbb{R}^{m \times m}$ are the inductance and resistance matrices, and $u \in \mathbb{R}^{n}$ is the injected current in each node.

Let us assume constant reference values and the Droop Control based on passivity-based techniques reported in [11] that ensures stabilisation of the voltage nodes, $e$, to $v$. The closed loop dynamics results in

$$
\begin{aligned}
\boldsymbol{C} \dot{e} & =-\boldsymbol{K}(e-v)-\boldsymbol{B} i+\boldsymbol{W} v \\
\boldsymbol{L} \dot{i} & =-\boldsymbol{R} i+\boldsymbol{B}^{T} e .
\end{aligned}
$$

From the network dynamics $(39)-(40)$, and using $\boldsymbol{G}=\boldsymbol{R}^{-1}$ and (2), one gets the supplied current in steady state,

$$
u_{s}=\boldsymbol{W} v
$$




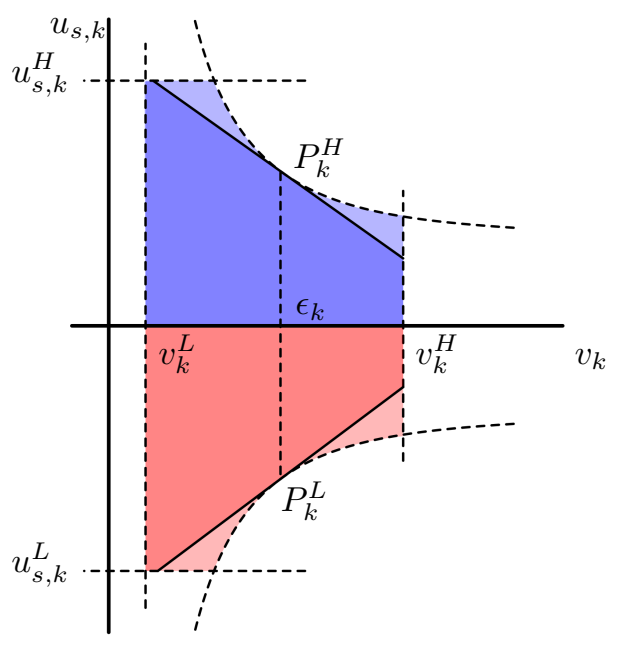

Figure 4: Admissibility region: power injection (blue); power consumption (red), and its linear approximation around $v_{o, k}$.

On the other hand, in DC circuits, the electrical power supplied/consumed by a node $k$ is given by $P_{k}=e_{k} u_{k}$ which, in steady-state, (i.e., $e=v$ and $u=u_{s}$ ) and using 43

$$
P_{k}(v)=v_{k}[\boldsymbol{W}]_{k} v
$$

where $[\boldsymbol{W}]_{k}$ denotes the $k t h$-row of matrix $\boldsymbol{W}$.

In a realistic operation, at any node $k$, each voltage/current pair $\left(e_{k}, u_{k}\right)$ must remain within an admissibility region and, consequently, the reference values too. This region corresponds to the closed area limited by the maximum and minimum allowed voltages, $v_{k}^{H}$ and $v_{k}^{L}$, the rated power of the current source, $P_{k}^{H}$ and $P_{k}^{L}$, and the current boundary values, $u_{s, k}^{H}$ and $u_{s, k}^{L}$, see Figure 4. Notice that $v_{k}^{H} \geq v_{k}^{L}>0$, while $P_{k}^{H} \geq 0$ and $P_{k}^{L} \leq 0$ stand for injecting and consuming modes, respectively.

\subsection{OPF problem statement for a multi-terminal DC network}

The OPF problem for the MTDC network consists of finding the optimal (minimal losses) point subject to the constraints on the desired node voltages, $v$, supplied/consumed powers, $P$, and the injected currents, $u_{s}$ shown in Figure 
4. Thus, the OPF can be defined by

$$
\begin{array}{cl}
\underset{v}{\min } & f(v)=v^{T} \boldsymbol{W} v \\
\text { s.t. } & \boldsymbol{A}_{v} v-b_{v}=0 \\
& \boldsymbol{A}_{u} u_{s}-b_{u}=0 \\
& \boldsymbol{A}_{P} P-b_{P}=0 \\
& \boldsymbol{Q}_{v}^{H} v-v^{H} \leq 0 \\
& v^{L}-\boldsymbol{Q}_{v}^{L} v \leq 0 \\
& \boldsymbol{Q}_{u}^{H} u_{s}-u^{H} \leq 0 \\
& u^{L}-\boldsymbol{Q}_{u}^{L} u \leq 0 \\
& \boldsymbol{Q}_{P}^{H} P-P^{H} \leq 0 \\
& P^{L}-\boldsymbol{Q}_{P}^{L} P \leq 0
\end{array}
$$

where, super-indices $H, L$ refer to the constrained higher and lower values. The matrices $\boldsymbol{A}_{(\cdot)} \in \mathbb{R}^{n_{(\cdot)} \times n}$ and $\boldsymbol{Q}_{(\cdot)}^{(\cdot)} \in \mathbb{R}^{n_{(\cdot)}^{(\cdot)} \times n}$ have $n$ columns, one for each node, and each of their rows identifies a node by having all 0 values except a 1 in the column corresponding to the node.

The proposed OPF algorithm in (24)-(26) assumes that both equality and inequality constraints are linear but, from (44), $P_{k}$, for $k=1, \ldots, n$, is not convex and nonlinear, nor the contraints in (48), (53) and (54) are convex. In order to apply the results obtained in Section 4 , the following linear approximation is used for (48) (the linear approximation of constraints in (53) and (54) can be obtained straightforwardly)

$$
P_{k_{j}}(v)-\left[b_{P}\right]_{j} \approx[\boldsymbol{N}]_{j}\left(v-\epsilon_{j}\right)
$$

for each $j \in\left\{1, \ldots, n_{v}\right\}$, where $\epsilon_{j}$ is the linearisation points for constraint $j$ (see Figure 4 $4, k_{j}$ denotes the node that corresponds to constraint $j$, and

$$
[\boldsymbol{N}]_{j}=\left.\nabla P_{j}\right|_{\epsilon_{j}} ^{T}=\left[\epsilon_{j}\right]_{k_{j}} \cdot[\boldsymbol{W}]_{k_{j}}+\left[\boldsymbol{W} \epsilon_{j}\right]_{k_{j}} \cdot[\boldsymbol{I}]_{k_{j}} .
$$

As $\epsilon_{j}$ must satify $P_{k_{j}}\left(\epsilon_{j}\right)=\left[b_{P}\right]_{j},[\boldsymbol{N}]_{j} \epsilon_{j}=2\left[b_{P}\right]_{j}$, and $[55$ can be written as

$$
P_{k_{j}}(v)-\left[b_{P}\right]_{j} \approx[\boldsymbol{N}]_{j} v-2 \cdot\left[b_{P}\right]_{j} .
$$

Notice that if $\left[\epsilon_{j}\right]_{k_{j}} \neq 0,\left[\boldsymbol{W} \epsilon_{j}\right]_{k_{j}}=\left[b_{P}\right]_{j} /\left[\epsilon_{j}\right]_{k_{j}}$ and then $\left[\epsilon_{j}\right]_{k_{j}}$ is the only coordinate of the point $\epsilon_{j}$ needed to determine the linear approximation of $P_{k_{j}}(v)-\left[b_{P}\right]_{j}$ and, from 56

$$
P_{k_{j}}^{L i n}(v)=[\boldsymbol{N}]_{j} v-\left[b_{P}\right]_{j}=\left[\epsilon_{j}\right]_{k_{j}} \cdot[\boldsymbol{W}]_{k_{j}} v+\frac{\left[b_{P}\right]_{j}}{\left[\epsilon_{j}\right]_{k_{j}}} \cdot v_{k_{j}}-\left[b_{P}\right]_{j} .
$$

Then, when $\left[b_{P}\right]_{j} \neq 0$, it is possible to compute the error of the linear approximation (57), namely $\operatorname{err}_{j}$, defined as the maximum of the values

$$
\frac{P_{k_{j}}^{L i n}(v)}{\left[b_{P}\right]_{j}}-1
$$


where $v_{k_{j}} \in\left[v_{k_{j}}^{L}, v_{k_{j}}^{H}\right],[\boldsymbol{W}]_{k_{j}} v \in\left[u_{k_{j}}^{L}, u_{k_{j}}^{H}\right]$ and $v_{k_{j}}[\boldsymbol{W}]_{k_{j}} v=\left[b_{P}\right]_{j}$. The linearisation point that minimize $\operatorname{err}_{j}$ is $\left[\epsilon_{j}\right]_{k_{j}}=\sqrt{v_{k_{j}}^{M} \cdot v_{k_{j}}^{H}}$ where

$$
v_{k_{j}}^{M}=\left\{\begin{array}{lll}
\max \left\{v_{k_{j}}^{L}, \frac{\left[b_{P}\right]_{j}}{u_{k_{j}}^{H}}\right\} & \text { if } & {\left[b_{P}\right]_{j}>0} \\
\max \left\{v_{k_{j}}^{L}, \frac{\left[b_{P}\right]_{j}}{u_{k_{j}}^{L}}\right\} & \text { if } & {\left[b_{P}\right]_{j}<0}
\end{array},\right.
$$

and

$$
\operatorname{err}_{j}=\frac{v_{k_{j}}^{M}+v_{k_{j}}^{H}}{\sqrt{v_{k_{j}}^{M} \cdot v_{k_{j}}^{H}}}-2 .
$$

Obviously, if $\left[b_{P}\right]_{j}=0$, then $P_{k_{j}}^{\text {Lin }}\left(v_{k_{j}}, u_{k_{j}}\right)=\left[\epsilon_{j}\right]_{k_{j}} \cdot u_{k_{j}}$ and $\operatorname{err}_{j}=0$.

Using (43) and (55), the OPF problem (45)-(54) can be written in a more compact form as (24)-26) with

$$
\boldsymbol{A}=\left(\begin{array}{c}
\boldsymbol{A}_{v} \\
\boldsymbol{A}_{u} \boldsymbol{W} \\
\boldsymbol{N}
\end{array}\right), \quad b=\left(\begin{array}{c}
b_{v} \\
b_{u} \\
2 b_{P}
\end{array}\right)
$$

and

$$
\boldsymbol{Q}=\left(\begin{array}{c}
\boldsymbol{Q}_{v}^{H} \\
\boldsymbol{Q}_{u}^{H} \boldsymbol{W} \\
\boldsymbol{N}^{H} \\
-\boldsymbol{Q}_{v}^{L} \\
-\boldsymbol{Q}_{u}^{L} \boldsymbol{W} \\
-\boldsymbol{N}^{L}
\end{array}\right), \quad d=\left(\begin{array}{c}
v^{H} \\
u^{H} \\
2 P^{H} \\
-v^{L} \\
-u^{L} \\
-2 P^{L}
\end{array}\right)
$$

where $\boldsymbol{N}^{L}, \boldsymbol{N}^{H}$ are analogously obtained as $\boldsymbol{N}$ in (56).

Since the rows in the matrix $\boldsymbol{Q}_{v}^{H}$ have all zeros except one 1, according to Proposition 3 , the OPF is asymptotically stable.

\subsection{Example: North Sea offshore wind integration network}

The electrical network considered for this example is the offshore wind integration grid in the North Sea 16, consisting in 19 lines and 19 nodes, which 9 are wind farms (WF), 5 are onshore grid stations (GS) with their corresponding $\mathrm{DC} / \mathrm{AC}$ converters, and 5 hubs (HUB) interconnecting WF and GS. Figure 5 shows the network structure.

The node parameters and the line lengths are shown in Tables 1 and 2 respectively, and the resistance per kilometer is $r=0.0195 \Omega / \mathrm{km}$. All the parame-

230 ters are obtained from [33. For all $k$ nodes, high and low voltage constraints are $v_{k}^{H}=265 \mathrm{kV}$ and $v_{k}^{L}=245 \mathrm{kV}$, respectively, and the current and power limits are given in Table 1. With the used parameters the linearisation error in 61) is, for all nodes, $0.085 \%$.

The OPF problem is solved with the barrier-based algorithm proposed in 235 (31). The $\boldsymbol{W}$ matrix in $(27)$ is calculated from the network topology shown in 


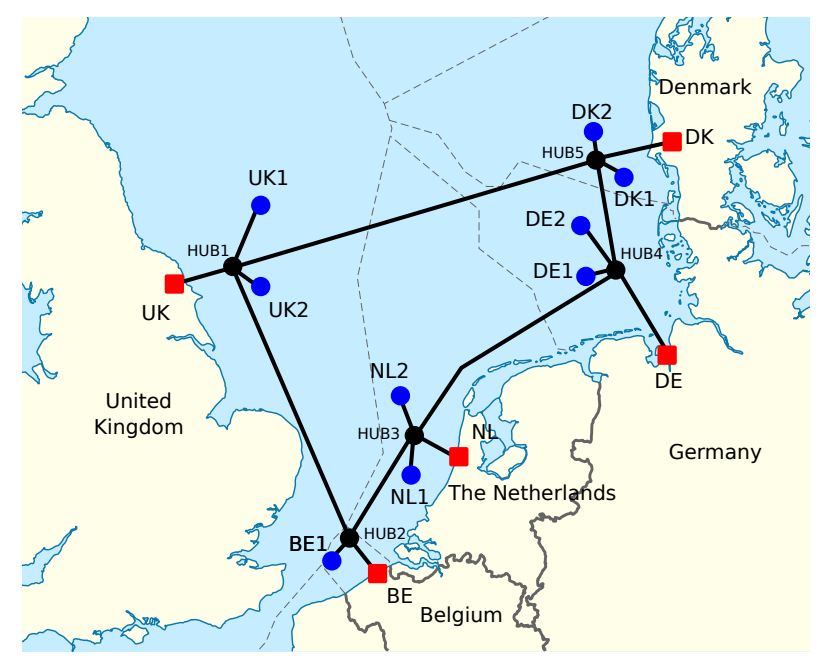

Figure 5: Scheme of the MTDC network representing the North Sea offshore wind integration used as testbench.

Figure 5, the resistance value $r$, and the line values in Table 2, The equality constraints in (28) are used to set the desired values for the power in the GS nodes (see Table 3 ) and to set the injected currents in the Hub nodes to zero (Hubs do not supply energy to the network). Then, $\boldsymbol{A}_{v}, b_{v}$ are empty, $\boldsymbol{A}_{u}$ is defined by 0 and 1 accordingly, $b_{u}=(0,0,0,0,0), \boldsymbol{N}$ is built according to (56) and $b_{P}$ take the values from Table 3 . The $\boldsymbol{A}$ matrix is built as $(62)$. The matrices $\boldsymbol{Q}_{v}^{(\cdot)}, \boldsymbol{Q}_{u}^{(\cdot)}$ and $\boldsymbol{N}^{(\cdot)}$ in $\boldsymbol{Q}$ representing, respectively, the node voltages, currents and power inequality constraints are defined as follows: $\boldsymbol{Q}_{v}^{(\cdot)}$ are identity matrices as all the node voltages are constrained; $\boldsymbol{Q}_{u}^{(\cdot)}$ are defined accordingly taking into account if the node current is constrained by higher or lower values as shown in columns $u^{H}$ and $u^{L}$ of Table 1 ; and $\boldsymbol{N}^{(\cdot)}$ are defined as explained in (56), taking into account if the node power is constrained by higher or lower values as shown in columns $P^{H}$ and $P^{L}$ of Table 1 .

\subsubsection{Implementation of the $O P F$}

The OPF algorithm is implemented using Matlab and has been running in a $2.3 \mathrm{GHz}$ Intel Core i5 microprocessor with a sampling time of $T_{s}=0.02 \mathrm{~s}$. The OPF parameters have been set as follows: $\boldsymbol{T}_{v}=5 \cdot 10^{-1} \cdot \boldsymbol{I}_{n} ; \boldsymbol{T}_{\lambda}=\operatorname{diagonal}\left(T_{\lambda, 1}\right.$. $\left.[\boldsymbol{A}]_{1} \cdot[\boldsymbol{A}]_{1}^{T}, \ldots, T_{\lambda, q} \cdot[\boldsymbol{A}]_{q} \cdot[\boldsymbol{A}]_{q}^{T}\right)$ where $T_{\lambda, j}$ is set to $10^{-2}$ or 0.5 depending on the equality constraint $j$ is, respectively a voltage, current or power constraint; and finally, $k_{j},(j=1, \ldots, p)$ is set to $10^{-2}$ or 1 depending on the inequality constraint $j$ is respectively a voltage, current or power constraint.

The test consists of testing the OPF algorithm by changing the power demanded from the GS nodes according to the values in Table 3. At $t=10 \mathrm{~s}$, the power demanded by N3 (GS in UK) drastically decreases from $70 \%$ to $25 \%$ and, 


\begin{tabular}{|c|c|c|c|c|c|c|}
\hline Node & Name & Type & $P_{k}^{H}$ & $P_{k}^{L}$ & $u_{k}^{H}$ & $u_{k}^{L}$ \\
\hline N1 & UK1 & WF & $600 \mathrm{MW}$ & - & $2.4 \mathrm{kA}$ & $0 \mathrm{kA}$ \\
N2 & UK2 & WF & $400 \mathrm{MW}$ & - & $1.6 \mathrm{kA}$ & $0 \mathrm{kA}$ \\
N3 & UK & GS & $850 \mathrm{MW}$ & $-850 \mathrm{MW}$ & $3.4 \mathrm{kA}$ & $-3.4 \mathrm{kA}$ \\
N4 & HUB1 & HUB & - & - & - & - \\
N5 & BE1 & WF & $200 \mathrm{MW}$ & - & $0.8 \mathrm{kA}$ & $0 \mathrm{kA}$ \\
N6 & BE & GS & $140 \mathrm{MW}$ & $-140 \mathrm{MW}$ & $0.56 \mathrm{kA}$ & $-0.56 \mathrm{kA}$ \\
N7 & HUB2 & HUB & - & - & - & - \\
N8 & NL1 & WF & $400 \mathrm{MW}$ & - & $1.6 \mathrm{kA}$ & $0 \mathrm{kA}$ \\
N9 & NL2 & WF & $200 \mathrm{MW}$ & - & $0.8 \mathrm{kA}$ & $0 \mathrm{kA}$ \\
N10 & NL & GS & $540 \mathrm{MW}$ & $-540 \mathrm{MW}$ & $2.16 \mathrm{kA}$ & $-2.16 \mathrm{kA}$ \\
N11 & HUB3 & HUB & - & - & - & - \\
N12 & DE1 & WF & $400 \mathrm{MW}$ & - & $1.6 \mathrm{kA}$ & $0 \mathrm{kA}$ \\
N13 & DE2 & WF & $400 \mathrm{MW}$ & - & $1.6 \mathrm{kA}$ & $0 \mathrm{kA}$ \\
N14 & DE & GS & $640 \mathrm{MW}$ & $-640 \mathrm{MW}$ & $2.56 \mathrm{kA}$ & $-2.56 \mathrm{kA}$ \\
N15 & HUB4 & HUB & - & - & - & - \\
N16 & DK1 & WF & $200 \mathrm{MW}$ & - & $0.8 \mathrm{kA}$ & $0 \mathrm{kA}$ \\
N17 & DK2 & WF & $200 \mathrm{MW}$ & - & $0.8 \mathrm{kA}$ & $0 \mathrm{kA}$ \\
N18 & DK & GS & $240 \mathrm{MW}$ & $-240 \mathrm{MW}$ & $0.96 \mathrm{kA}$ & $-0.96 \mathrm{kA}$ \\
N19 & HUB5 & HUB & $600 \mathrm{MW}$ & - & - & - \\
\hline
\end{tabular}

Table 1: North Sea integration grid: node parameters.

to $80 \%$. A second change occurs at $t=20 \mathrm{~s}$, when N6 (GS in Belgium) decreases from $50 \%$ to $25 \%$ and N10 and N18 (GS in The Netherlands and Denmark) increase up to $65 \%$ and $95 \%$, respectively.

Regarding the supervision and control scheme of Figure2 the OPF provides the required node voltages according to the above mentioned changes of power demands/generations, see Figure 6. Notice that the solutions remain within the limits $v_{k}^{H}, v_{k}^{L}$, which suggests the use of the trajectories obtained from the OPF as control inputs for the DC network. As expected, the voltages remain around the highest values (implying the power transmission with less current, minimizing the Joule's effect). One can also observe how the voltages in the WF nodes are higher than the ones in the GS nodes, allowing the current flow from WF to GS.

The power demanded by the OPF for each node is calculated using 444. The obtained power values in the GS nodes are depicted (in blue) in Figure 7 .

275 whose go to the desired values (in dotted black) without violating the power constraints (in dotted red).

The required powers of the WF nodes are also in the restricted area, see Figure 8. Notice how some WF nodes saturate: N2 from 0 to 10s, and N9 from 20 to 30 s, because the closest GS is demanding high power.

Figures 9, 10 and 11 show the demanded node currents. All the currents are between the admissible values (Figures 9 and 10) and the solution for the Hub currents converges to zero, see Figure 11. 


\begin{tabular}{|c|c|c|c|}
\hline Line & Length [km] & Line & Length $[\mathrm{km}]$ \\
\hline $\mathrm{L}_{1,4}$ & 100 & $\mathrm{~L}_{11,15}$ & 250 \\
$\mathrm{~L}_{2,4}$ & 40 & $\mathrm{~L}_{12,15}$ & 40 \\
$\mathrm{~L}_{3,4}$ & 120 & $\mathrm{~L}_{13,15}$ & 70 \\
$\mathrm{~L}_{4,7}$ & 300 & $\mathrm{~L}_{14,15}$ & 150 \\
$\mathrm{~L}_{5,7}$ & 50 & $\mathrm{~L}_{15,19}$ & 120 \\
$\mathrm{~L}_{6,7}$ & 100 & $\mathrm{~L}_{16,19}$ & 40 \\
$\mathrm{~L}_{7,11}$ & 120 & $\mathrm{~L}_{17,19}$ & 50 \\
$\mathrm{~L}_{8,11}$ & 100 & $\mathrm{~L}_{18,19}$ & 150 \\
$\mathrm{~L}_{9,11}$ & 40 & $\mathrm{~L}_{1,19}$ & 380 \\
$\mathrm{~L}_{10,11}$ & 70 & - & - \\
\hline
\end{tabular}

Table 2: North Sea integration grid: line parameters.

\begin{tabular}{|c|c|c|c|c|c|}
\hline Node & Name & Type & $t=0 \mathrm{~s}$ & $t=10 \mathrm{~s}$ & $t=20 \mathrm{~s}$ \\
\hline N3 & UK & GS & $70 \%$ & $25 \%$ & $25 \%$ \\
N6 & BE & GS & $50 \%$ & $50 \%$ & $25 \%$ \\
N10 & NL & GS & $40 \%$ & $40 \%$ & $65 \%$ \\
N14 & DE & GS & $30 \%$ & $80 \%$ & $80 \%$ \\
N18 & DK & GS & $50 \%$ & $50 \%$ & $95 \%$ \\
\hline
\end{tabular}

Table 3: Numerical test: \% of the demanded power.

Finally, Figure 12 shows the computer processing time (in \% with respect to the sampling time). It can be noticed that always remain below $25 \%$.

5.3.2. Comparison of the continuous-time with respect to the classical approach

As mentioned in the introduction, one of the main interests of using the continuous-time gradient method is the possibility of using the instantaneous value resulting from OPF algorithm as the input of the MTDC network. To this end, a simulation test has been carried out by connecting the OPF with a model emulating the dynamics of the North Sea HVDC network. In 21, has been proved that the cascade interconnection of the primal-dual dynamics (34) with a DC network is asymptotically stable. The same scenario in the previous test has been used, and the resulting desired node voltages have been used as a reference for the droop voltage controller presented in [11. The parameters for the network dynamics are: inductance per kilometer $l=19 \mathrm{mH} / \mathrm{km}$, and, in all nodes the capacitances are $C_{k}=75 \mu \mathrm{F}$.

Figure 13 (top) shows the error between the provided values by the OPF algorithm, $v$, and the controlled voltages of the DC network, e. As expected, the error of the voltage values tends to be zero, with a maximum transient error of $0.6 \mathrm{kV}$.

Usually, the OPF algorithms provide the optimal values when they reach a value with a certain tolerance. This implies that the solution is only available after the computation time required by the method, every $T_{\mathrm{OPF}}$ seconds, see example in [16. This section compares the behaviour of the MTDC network 


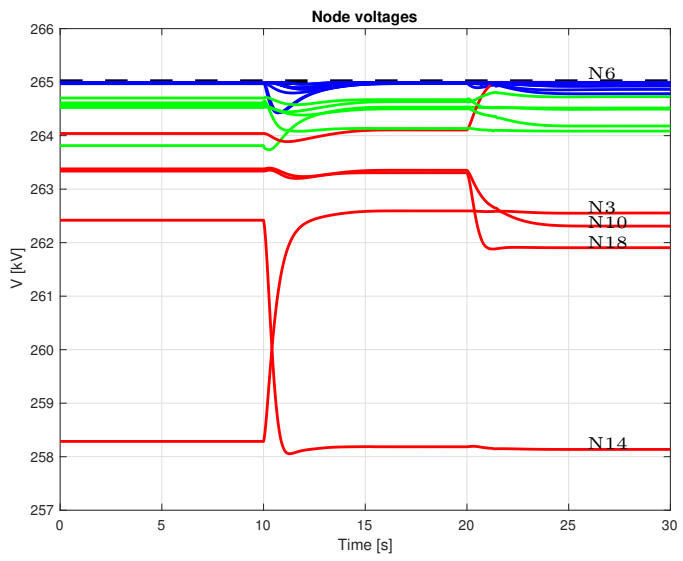

Figure 6: OPF implementation results: grid voltages. Grid side (GS) in red, Wind farms (WF) in blue, and Hub in green. GS nodes are labeled.

305

using a continuous-time OPF with the classical approach. In the first case, the OPF output is the trajectory solution of (31) and, in the second case, by sampling the solution of (31) each $T_{\mathrm{OPF}}=5 \mathrm{~s}$.

The simulation results are shown in Figure 13 (middle and bottom). With the classical approach the maximum errors are considerably higher (almost 5 $\mathrm{kV}$ ), see Figure 13 (middle). Finally, the voltage of node N3 for both cases is depicted as an illustrative example in Figure 13 (bottom). The benefits of using the continuous-time approach are: the optimal value is reached by the real network sooner (of course, depending on $T_{\mathrm{OPF}}$ ) and the overshoot is lower because the reference value is continuous and slowly ${ }^{1}$ varying.

\section{Conclusions}

The OPF problem for a DC network has been written using the portHamiltonian formalism. The main feature of this description is the ability of interconnecting dynamics preserving the stability properties. In this paper it has been show that the gradient method applied to the OPF problem for minimizing losses in DC networks is stable under the conditions of the constraints $\mathrm{C} 1$ and $\mathrm{C} 2$ given in Proposition 3. The paper also includes the case of constrained problems by using barrier functions that prevent solutions out of the admissibility region.

The behaviour of the OPF algorithm is illustrated with the North Sea wind integration network. The numerical results show the benefit of using a continuous-time algorithm that is a possibility of integrating the OPF algorithm

\footnotetext{
${ }^{1}$ Note that the speed of convergence of the OPF is tuneable using the parameters $\boldsymbol{T}_{v}, \boldsymbol{T}_{\lambda}$.
} 

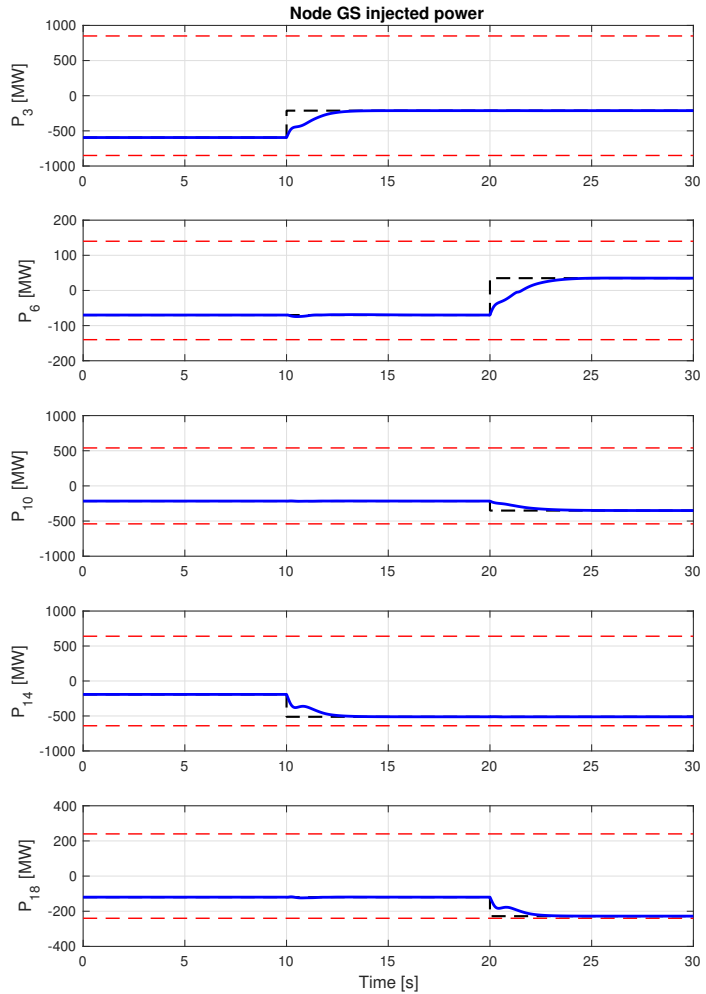

Figure 7: OPF implementation results: Grid side (GS) injected powers. Limit powers (in red), reference power (dotted black) and OPF result (in blue).

with the network dynamics and treating the supervision and control problem as a whole, regulating the voltages of the DC network while the OPF algorithm searches the optimal point. Consequently, and in contrast with the traditional schemes where the OPF works with a certain sampling time, the method presented in this paper allows a faster regulation and smaller overshoots because the (continuous) trajectories resulting from the OPF search are used as inputs for the network controller.

\section{Acknowledgments}

D. del Puerto Flores was supported, in part, by the internal project PROSNI2018 and the Mexican PRODEP project UDG-PTC-1319. The work of A. DòriaCerezo was partially supported by the Government of Spain through the Agencia 

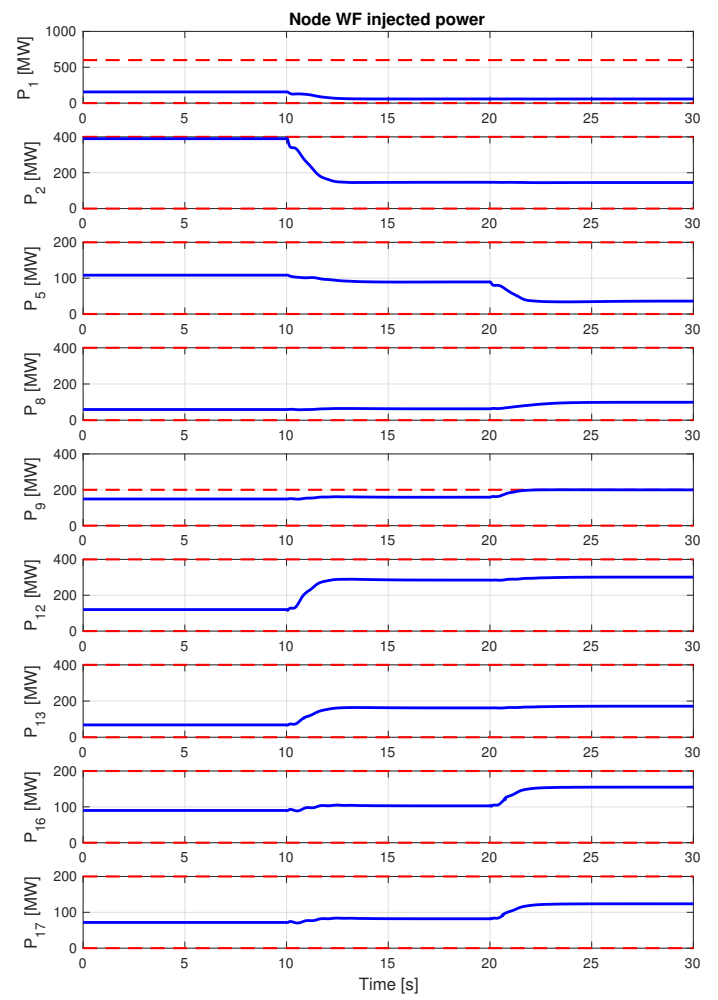

Figure 8: OPF implementation results: Wind farm (WF) injected powers. Limit powers (in red) and OPF result (in blue).

Estatal de Investigación Project DPI2017-85404-P and by the Generalitat de Catalunya through the Project 2017 SGR 872.

\section{References}

[1] A. Shivakumar, B. Normark, M. Welsch, Household DC networks: State of the art and future prospects, InsightE Rapid Response Energy Brief (2015) $1-11$.

[2] D. van Hertem, M. Ghandhari, Multi-terminal VSC HVDC for the European supergrid: Obstacles, Renewable \& Sustainable Energy Reviews 14 (9) (2010) 3156-3163.

[3] A. T. Elsayed, A. A. Mohamed, O. A. Mohammed, DC microgrids and 

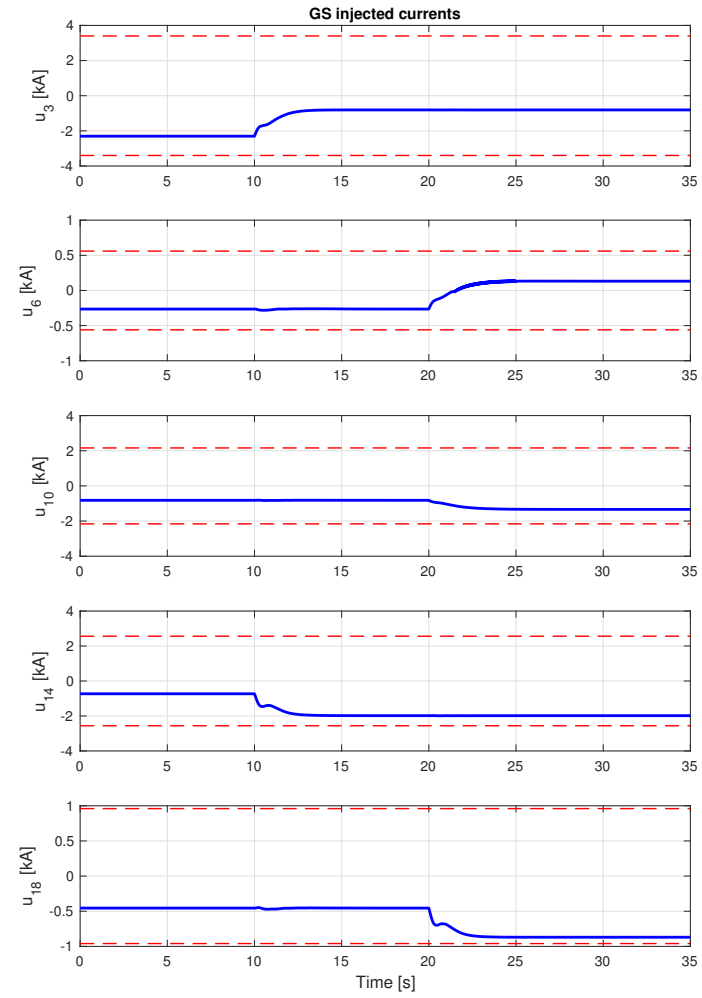

Figure 9: OPF implementation results: Grid side (GS) injected currents. Limit currents (in red) and OPF result (in blue).

distribution systems: An overview, Electric Power Systems Research 119 (2015) 407-417.

[4] E. Prieto-Araujo, F. Bianchi, A. Junyent-Ferré, O. Gomis-Bellmunt, Methodology for droop control dynamic analysis of multiterminal VSCHVDC grids for offshore wind farms, IEEE Trans. on Power Delivery 26 (4) (2011) 2476-2485.

[5] C. Gavriluta, I. Candela, J. Rocabert, A. Luna, P. Rodríguez, Adaptive droop for control of multiterminal DC bus integrating energy storage, IEEE Trans. on Power Delivery 30 (1) (2015) 16-24.

[6] W.W. Weaver, R.D. Robinett III, G.G. Parker, D.G. Wilson, Distributed control and energy storage requirements of networked DC microgrids, Control Engineering Practice 44 (2015) 10-19. 


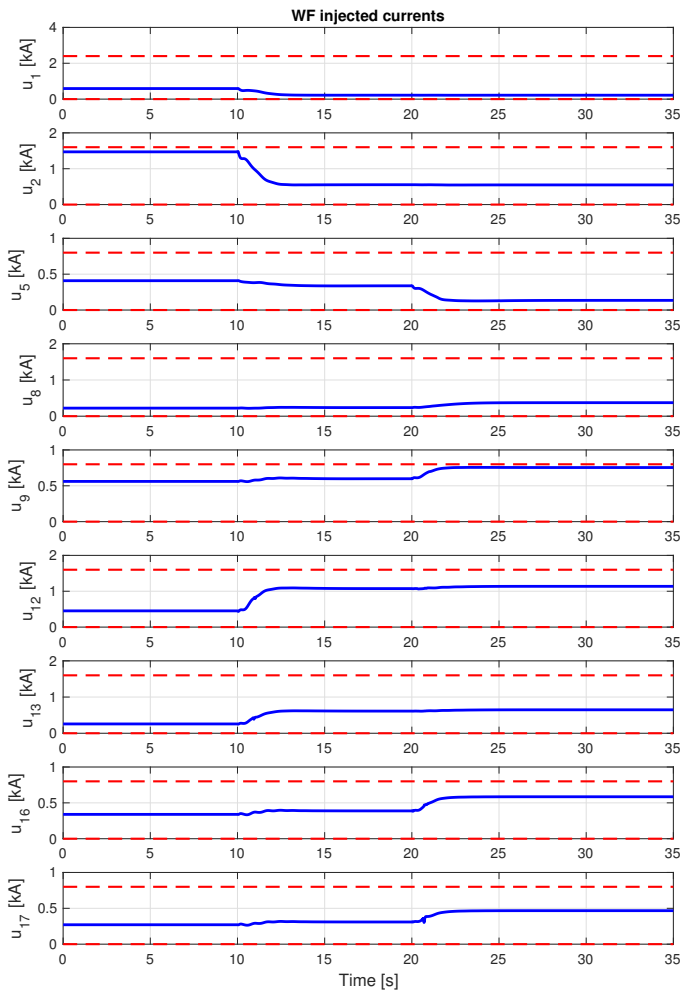

Figure 10: OPF implementation results: Wind farm (WF) injected currents. Limit currents (in red) and OPF result (in blue).

[7] Y. Li, F. Liu, Y. Cao, Delay-dependent wide-area damping control for stability enhancement of HVDC/AC interconnected power systems, Control Engineering Practice 37 (2016) 43-54.

[8] P. Mc Namara, R. Negenborn, B. de Schutter, G. Lightbody, S. Mc Loone, Distributed MPC for frequency regulation in multi-terminal HVDC grids, Control Engineering Practice 46 (2016) 176-187.

[9] P. Mc Namara, R. Meere, T. O'Donnell, S. McLoone, Control strategies for automatic generation control over MTDC grids, Control Engineering Practice 54 (2016) 129-139.

[10] D. Zonetti, R. Ortega, A. Benchaib, Modeling and control of HVDC transmission systems from theory to practice and back, Control Engineering Practice 45 (2015) 133-146. 


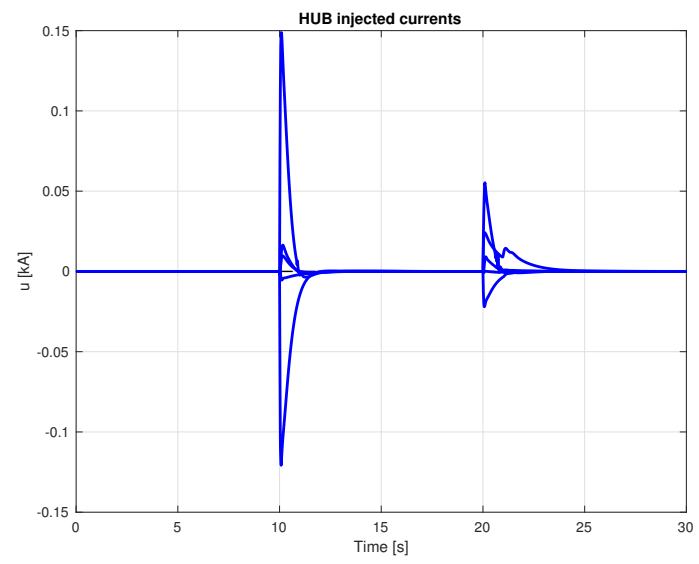

Figure 11: OPF implementation results: Hub injected currents.

[11] A. Dòria-Cerezo, J.M. Olm, J.M.A. Scherpen, Passivity-based control of multi-terminal HVDC systems under control saturation constraints, in: Proc. 5th IFAC Workshop in Lagrangian and Hamiltonian Methods for Non Linear Control, 2015.

[12] B. Yang, Y. Sang, K. Shi, W. Yao, L. Jiang, T. Yu, Design and real-time implementation of perturbation observer based sliding-mode control for VSC-HVDC systems, Control Engineering Practice 56 (2016) 13-26.

[13] A. Dòria-Cerezo, J.M. Olm, M. di Bernardo, E. Nuño, Modelling and control for bounded synchronization in multi-terminal VSC-HVDC transmission networks, IEEE Trans. on Circuits and Systems-I 63 (3) (2016) 916925.

[14] K. Rouzbehi, J. Candela, G. Gharehpetian, L. Harnefors, A. Luna, P. Rodriguez, Multiterminal DC grids: Operating analogies to AC power systems, Renewable \& Sustainable Energy Reviews Sustainable Energy Reviews 70 (2017) 886-895.

[15] M. Aragüés-Peñalba, A. Egea-Àlvarez, O. Gomis-Bellmunt, A. Sumper, Optimum voltage control for loss minimization in HVDC multi-terminal transmission systems for large offshore wind farms, Electric Power Systems Research 89 (2012) 54-63.

[16] S. Rodrigues, R. Teixeira-Pinto, P. Bauer, J. Pierik, Optimal power flow control of VSC-based multiterminal DC networks offshore wind integration in the North Sea, IEEE Journal of Emerging and Selected Topics in Power Electronics 1 (4) (2013) 260-268. 


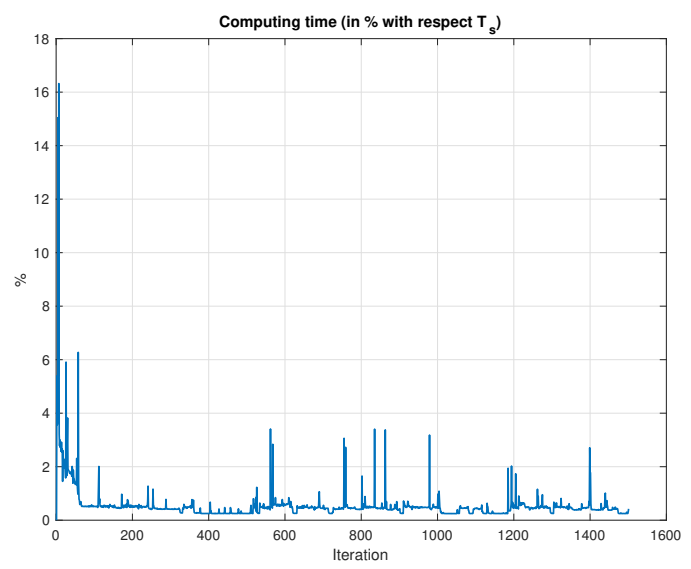

Figure 12: OPF implementation results: computer processing time (in $\%$ with respect to the sampling time).

[17] Z. Shuai, J. Fang, F. Ning, Z. Shen, Hierarchical structure and bus voltage control of DC microgrid, Renewable \& Sustainable Energy Reviews 82 (2018) 3670-3682.

[18] C. Gavriluta, I. Candela, A. Luna, A. Gómez-Expósito, P. Rodríguez, Hierarchical control of HV-MTDC systems with droop-based primary and OPF-based secondary, IEEE Trans. on Smart Grid 6 (3) (2015) 1502-1510.

[19] C. Gavriluta, R. Caire, A. Gomez-Exposito, N. Hadjsaid, A distributed approach for OPF-based secondary control of MTDC systems, IEEE Trans. on Smart Grid (In press) 1-9.

[20] E. Benedito, D. del Puerto-Flores, A. Dòria-Cerezo, J.M.A. Scherpen, Optimal power flow for resistive DC networks: a port-Hamiltonian approach, IFAC-PapersOnLine 50 (1) (2017) 25-30.

[21] E. Benedito, D. del Puerto-Flores, O. van der Feltz, A. Dòria-Cerezo, J.M.A. Scherpen, The gradient method for minimization loses in DC networks: passivity properties and interconnection, Submitted to IEEE Trans. on Control of Network Systems (Under review) -.

[22] L. Gan, S. Low, Optimal power flow in direct current networks, IEEE Trans. on Power Systems 29 (6) (2014) 2892-2904.

[23] J. Li, F. Liu, Z. Wang, S. Low, S. Mei, Optimal power flow in stand-alone DC microgrids, arXiv preprint. arXiv:1708.05140v1 (2018) 1-12.

[24] A. Cherukuri, J. Cortés, Asymptotic stability of saddle points under the saddle-point dynamics, in: Proc. American Control Conference 2015, 2015. 

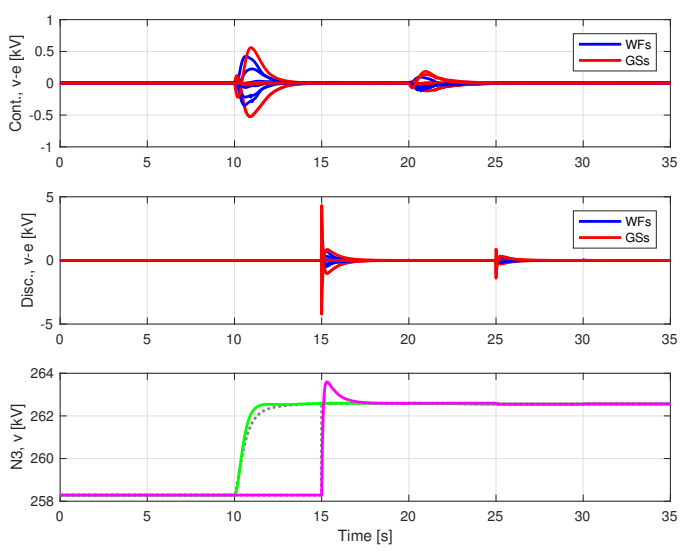

Figure 13: Simulation results of the OPF connected with a controlled DC network. Top: voltage errors using the continuous solution provided by the OPF; Middle: voltage errors using a sample time, $T_{\mathrm{OPF}}$, of $5 \mathrm{~s}$; Bottom: real voltage in node $\mathrm{N} 3$ when the OPF is implemented in a continuous (green) or discrete (magenta) time.

[25] T. Stegink, C. de Persis, A. van der Schaft, A unifying energy-based approach to stability of power grids with market dynamics, IEEE Trans. on Automatic Control 62 (6) (2017) 2612-2622.

[26] D. Feijer, F. Paganini, Stability of primal-dual gradient dynamics and applications to network optimization, Automatica 46 (2010) 1974-1981.

[27] T.W. Stegink, C. de Persis, A.J. van der Schaft, Port-Hamiltonian formulation of the gradient method applied to smart grids, in: Proc. 5th IFAC Workshop in Lagrangian and Hamiltonian Methods for Non Linear Control, 2015.

[28] N. Biggs, Algebraic Graph Theory, Cambridge University Press, Cambride, UK, 1974.

[29] A. van der Schaft, Characterization and partial synthesis of the behavior of resistive circuits at their terminals, Systems \& Control Letters 59 (7) (2010) 423-428.

[30] J. Arrow, L. Hurwicz, H. Uzawa, H. Chenery, Studies in linear and nonlinear programming, Stanford University Press, 1958.

[31] E. Benedito, D. del Puerto-Flores, A. Dòria-Cerezo, O. van der Feltz, J.M.A. Scherpen, Strictly convex loss functions for port-Hamiltonian based optimization algorithm for MTDC networks, in: Proc. 55th Conference on Decision and Control, 2016.

[32] S. Boyd, L. Vandenberghe, Convex optimization, Cambridge University Press, 2004. 
[33] R. Teixeira-Pinto, Multi-terminal DC networks system integration, dynamics and control, Ph.D. thesis, Technische Universiteit Delft (2014). 